\title{
Receiver Implementation for MIMO-OFDM with AMC and Precoding
}

\author{
Johanna Ketonen $\dagger$ and Markku Juntti $\dagger$ \\ Centre for Wireless Communications \\ P.O. Box 4500, FIN-90014 University of Oulu, Finland \\ \{johanna.ketonen, markku.juntti\}@ee.oulu.fi
}

\author{
Joseph R. Cavallaro* \\ Dept. of Electrical \& Computer Engineering \\ Rice University, Houston, TX 77251-1892, USA \\ cavallar@rice.edu
}

\begin{abstract}
Receivers for horizontally encoded LTE based MIMO-OFDM systems are considered in this paper. Adaptive modulation and coding (AMC) is used as well as precoding. The linear minimum mean square error (LMMSE), successive interference cancellation (SIC) and $K$-best list sphere detectors (LSD) are compared. The receivers were designed and implemented for $2 \times 2$ and $4 \times 4$ antenna systems and meet the decoding rate requirement in LTE, i.e, $210 \mathrm{Mb} / \mathrm{s}$ in $2 \times 2$ and $405 \mathrm{Mb} / \mathrm{s}$ in $4 \times 4$ antenna systems. The results show that the performance of the receivers is similar in low SNR but the performance difference increases when a higher rank transmission is used. The $K$-best LSD has the highest performance and complexity. A simpler receiver could be used in the low SNRs to save power and a more complex receiver in the high SNRs when a higher goodput is needed.
\end{abstract}

\section{INTRODUCTION}

Multiple-input multiple-output (MIMO) systems offer an increase in capacity or diversity. Orthogonal frequency division multiplexing (OFDM) is a popular technique for wireless high data-rate transmission because it enables efficient use of the available bandwidth and a simple implementation. The combination of MIMO and OFDM is a promising wireless access scheme [1] and it has been adopted in the third generation (3G) long term evolution (LTE) standard. LTE includes a downlink transmitter structure, where the data is divided into two streams which are encoded separately [2]. Precoding is also included in the LTE specifications.

Sphere detectors calculate the maximum likelihood (ML) solution by taking into account the lattice points that are inside a sphere of a given radius [3]. List sphere detectors (LSD) approximate the maximum a posteriori probability (MAP) detector and provide soft outputs for the forward error control (FEC) decoder [4]. The $K$-best LSD is a modification of the $K$-best algorithm [5].

Linear minimum mean square error (LMMSE) detection principles can be straightforwardly applied in MIMO detection. Ordered serial interference canceller (OSIC) was proposed already in the original papers considering the Bell Laboratories layered space-time (BLAST) architecture [6], [7] for

$\dagger$ This research was done in the MITSE project which was supported by Elektrobit, Nokia, Nokia Siemens Networks, Uninord and the Finnish Funding Agency for Technology and Innovation (TEKES).

*This work was supported in part by Nokia, Nokia Siemens Networks, Texas Instruments, Xilinx, and by the US National Science Foundation under grants CCF-0541363, CNS-0551692, CNS-0619767, EECS-0925942 and CNS-0923479. improving the performance of the LMMSE detection. Therein, instead of jointly detecting signals from all the antennas, the strongest signal can be detected first and its interference can be cancelled from each received signal. In FEC encoded systems, the detected symbols are decoded before cancellation. The performance of linear receivers with different code rates and SNRs was analyzed in [8].

In this paper, LMMSE, successive interference cancellation (SIC) and $K$-best LSD detectors are implemented and compared for a $2 \times 2$ and a $4 \times 4$ antenna system with adaptive modulation and coding (AMC). With AMC, the receiver has to be able to operate with all possible modulation schemes. Thus, the receivers are designed to process QPSK, 16-QAM and 64QAM. The decoding rates of the application-specific integrated circuit (ASIC) implementations are designed to be $210 \mathrm{Mb} / \mathrm{s}$ in the $2 \times 2$ antenna case and $405 \mathrm{Mb} / \mathrm{s}$ in the $4 \times 4$ antenna case, which is sufficient for the $3 \mathrm{G}$ LTE $20 \mathrm{MHz}$ bandwidth. The algorithms can be then compared in terms of performance and hardware complexity. As the transmission is adapted to the channel conditions, it is studied, if a simpler receiver can be used and a performance close to that of a more complex receiver can be achieved. The channel capacity calculation for channel-quality indication (CQI) is also implemented. The precoding complexity-performance tradeoff is also considered.

The paper is organized as follows. The system model is presented in Section II. The SIC algorithm is introduced in Section III. The $K$-best LSD algorithm is introduced in Section IV. Some performance examples are shown in Section V. Implementation results are presented in Section VI.

\section{SYSTEM MODEL}

An OFDM based MIMO transmission system with $N$ transmit (TX) and $M$ receive (RX) antennas, where $N \leq M$, is considered in this paper. A layered space-time architecture with horizontal encoding is applied, i.e, the two data streams are encoded and decoded separately. In the $4 \times 4$ antenna system, each of the two streams are multiplexed onto two antennas; the first stream is multiplexed onto the first and second antenna and the second stream onto the third and fourth antenna. The system model is shown in Figure 1. The received signal is detected jointly or separately and log-likelihood ratios (LLR) are calculated from the detected symbols.

The received signal can be described with the equation

$$
\mathbf{y}_{k}=\mathbf{H}_{k} \mathbf{P} \mathbf{x}_{k}+\eta_{k}, \quad k=1,2, \ldots, K,
$$




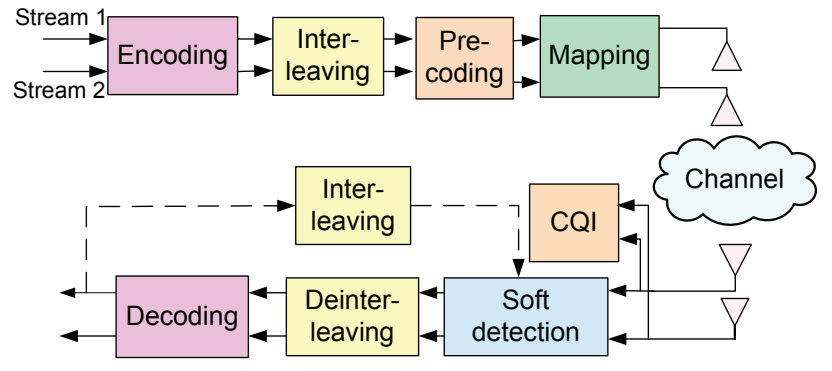

Fig. 1. The MIMO-OFDM system model in 3G LTE.

where $K$ is the number of subcarriers, $\mathbf{x}_{k} \in \mathbb{C}^{N \times 1}$ is the transmitted signal, $\eta_{k} \in \mathbb{C}^{M \times 1}$ is a vector containing identically distributed complex Gaussian noise, $\mathbf{P} \in \mathbb{C}^{r \times N}$ is the precoding matrix with rank $r$ and $\mathbf{H}_{k} \in \mathbb{C}^{M \times N}$ is the channel matrix containing complex Gaussian fading coefficients. The entries of $\mathbf{x}_{k}$ are from a complex QAM constellation $\Omega$ and $|\Omega|=2^{Q}$, where $Q$ is the number of bits per symbol. The set of possible transmitted symbol vectors is $\Omega^{N}$.

\section{A. AMC and precoding}

Feedback from the receiver to the transmitter can be used to improve the performance of the system. The channel capacity or signal-to-interference-noise ratio (SINR) based channel quality indicator (CQI) is calculated in the receiver and then sent back to the transmitter. The CQI includes the indices to adapt modulation, code rate and transmission rank for the downlink transmission. A suitable precoding matrix from a predefined codebook is also informed in the form of a precoding matrix indicator (PMI) [2]. The channel capacities with different precoding matrices are calculated in the receiver and the highest capacity achieving precoding matrix is selected. A lookup table is used to select the best modulation, code rate and transmission rank combination for the calculated capacity while maintaining the target frame error rate (FER).

The capacity can be calculated as

$$
C=\log \left(\operatorname{det}\left(\mathbf{I}+\frac{E_{s}}{\sigma^{2} M} \mathbf{H}^{\mathrm{H}} \mathbf{H}\right)\right)
$$

and it is summed over all the subcarriers in the frame to obtain the threshold used in the modulation and coding scheme (MCS) selection. If precoding is used, $\mathbf{H}$ in (2) is replaced by $\mathbf{P H}$ and the $\mathbf{P}$ with the largest capacity is selected. The instantaneous SINR can also be used in the MCS selection and it can be calculated for the $j$ th stream as

$$
\gamma_{j}=\frac{1}{\left[\left(\mathbf{I}+\frac{E_{s}}{\sigma^{2} M} \mathbf{H}^{\mathrm{H}} \mathbf{H}\right)^{-1}\right]_{j j}} .
$$

The capacity based MCS selection is assumed in this paper.

\section{The SIC ALGORITHM}

The soft SIC receiver is illustrated in Figure 2. The first layer is detected with an LMMSE detector. The LLR values are calculated from the LMMSE outputs. The de-interleaved stream is decoded with a turbo decoder and symbol expectations are calculated. The expectations are cancelled from the second layer. The first layer remains the same after the second iteration. The details of the SIC algorithm are described in [9].

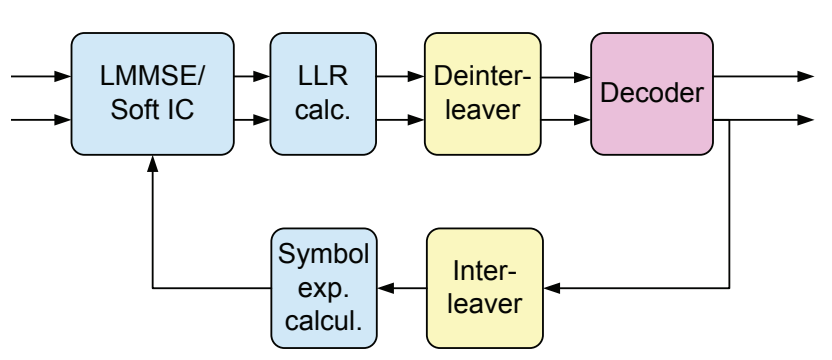

Fig. 2. The soft SIC receiver.

The weight matrix is calculated according to the MMSE rule

$$
\mathbf{W}=\left(\mathbf{H}^{\mathrm{H}} \mathbf{H}+\sigma^{2} \mathbf{I}_{M}\right)^{-1} \mathbf{H}^{\mathrm{H}},
$$

where $\mathbf{H}$ is the channel matrix, $\sigma^{2}$ is the noise variance, $(\cdot)^{\mathrm{H}}$ is the complex conjugate transpose and $\mathbf{I}_{M}$ is a $M \times M$ identity matrix. The layer for detection is chosen according to the postdetection signal-to-noise ratio (SNR) and the corresponding nulling vector is chosen from the weight matrix $\mathbf{W}$ [6]. All the weight matrices in an OFDM symbol are calculated and the layer to be detected is chosen according to the average over all the subcarriers.

The MMSE weight matrix can also be calculated from the extended channel matrix

with

$$
\mathbf{H}_{e x}=\left[\begin{array}{l}
\mathbf{H} \\
\sigma \mathbf{I}
\end{array}\right]
$$

$$
\mathbf{W}=\mathbf{R}^{-1} \mathbf{Q}^{\mathrm{H}}
$$

where $\mathbf{Q}$ and $\mathbf{R}$ are from $\mathrm{QRD}$ of the matrix $\mathbf{H}_{e x}$.

\section{THE K-BEST LSD ALGORITHM}

List sphere detectors (LSD) can be used to approximate the MAP detector and to provide soft outputs for the decoder [4]. The $K$-best algorithm [5] is a breadth-first search based algorithm, and keeps the $K$ nodes which have the smallest accumulated Euclidean distances at each level. A LSD structure is presented in Figure 3. The channel matrix $\mathbf{H}$ is first decomposed into matrices $\mathbf{Q}$ and $\mathbf{R}$ in the $\mathbf{Q R}$-decomposition block. The extended channel matrix is used in the QRD to improve the overall performance of the LSD. Euclidean distances between the received signal vector $\mathbf{y}$ and possible transmitted symbol vectors are calculated in the LSD block. The candidate symbol list is demapped to binary form. The log-likelihood ratios are calculated in the LLR block. They can be updated iteratively from the outputs of the turbo decoder as presented in [10].

\section{PERFORMANCE COMPARISON}

The performance of the receiver algorithms with AMC is presented in Figure 4 in a $2 \times 2$ antenna case. A $5 \mathrm{MHz}$ bandwidth was used and a correlated typical urban channel [11] with BS azimuth spread of 2 degrees was applied. The 


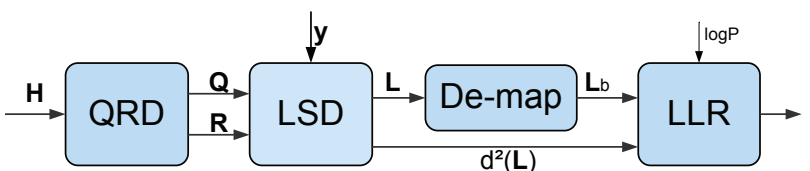

Fig. 3. The list sphere detector.

user velocity was $30 \mathrm{~km} / \mathrm{h}$. The modulations used in the AMC scheme were QPSK, 16-QAM and 64-QAM and the code rates were $1 / 3,1 / 2,2 / 3,3 / 4$ and $4 / 5$. The precoding matrices from [2] were used. Mostly rank 1 transmission was used in lower SNRs. When rank 2 transmission is used, the $K$-best LSD receiver with a list size of 8 performs the best and the LMMSE receiver has the worst performance. Updating the LLRs from the decoding improves the performance of the $K$-best by one dB.

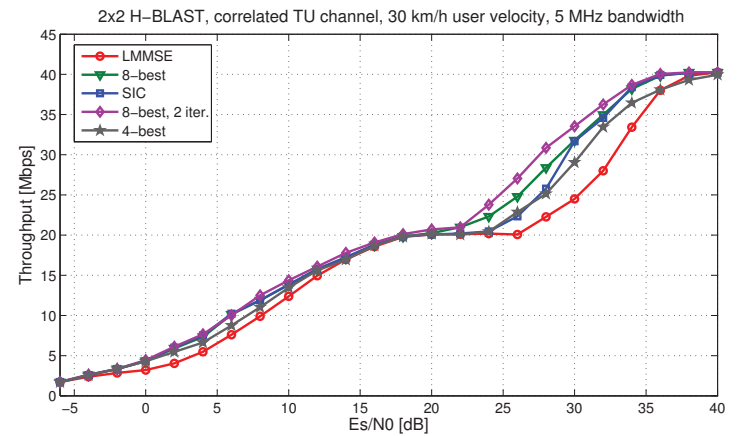

Fig. 4. $2 \times 2$ data transmission throughput vs. SNR in a correlated channel.

The performance of the algorithms in a channel with less correlation than in Figure 4 is shown in Figure 5. The difference in performance is smaller when the channel is better.

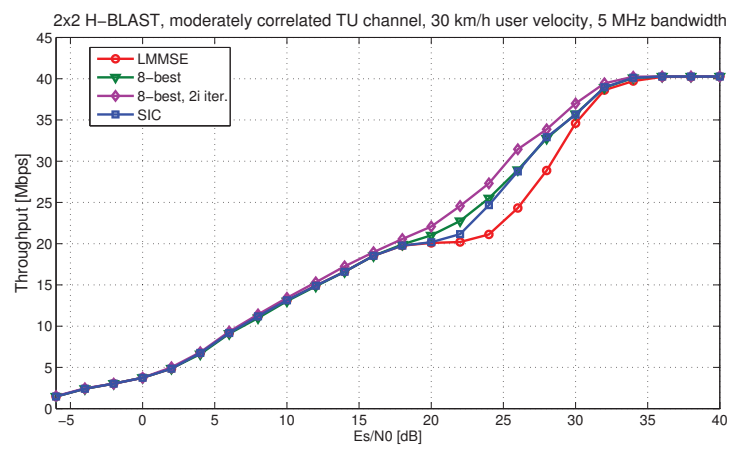

Fig. 5. $2 \times 2$ data transmission throughput vs. SNR in a moderately correlated channel.

The performance in a $4 \times 4$ antenna system is shown in Figure 6. The transmission rank of the precoding matrix was 1,2 or 4 . The performance of the algorithms is similar with rank 1 transmissions as in the $2 \times 2$ antenna system. With higher SNRs and transmission rank, the performance improves with the more complex receivers. The SIC receiver does not improve the performance much from the LMMSE in the high
SNRs when rank 4 transmission is used. This is partly due to the LTE encoding structure and the two strongest streams are cancelled from the other streams and only MMSE is used to separate the remaining streams.

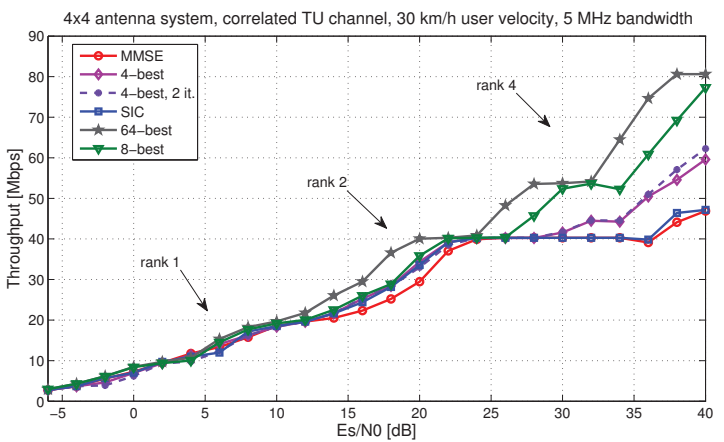

Fig. 6. $4 \times 4$ data transmission throughput vs. SNR with AMC and precoding.

The performance of the LMMSE receiver in a $4 \times 4$ system with different fixed modulation, coding schemes and transmission ranks is presented in Figure 7. The throughput with adaptive modulation and coding and precoding is also shown. Precoding improves the throughput from the fixed MCS upper bound up to $5 \mathrm{~dB}$. In the $2 \times 2$ antenna system, the performance is not greatly improved with precoding.

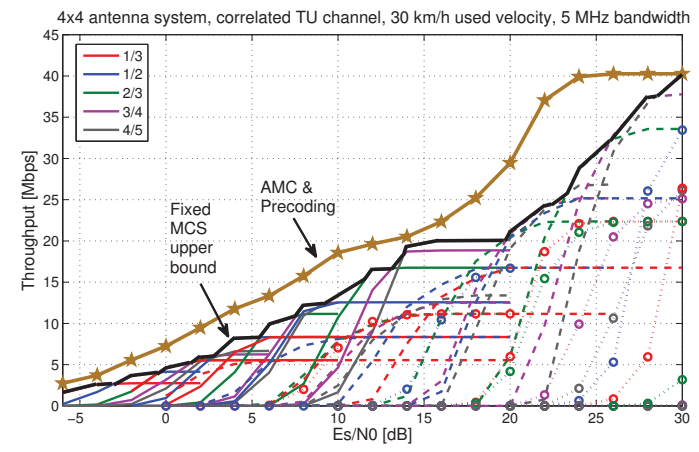

Fig. 7. Throughput with fixed MCSs and AMC with precoding.

\section{ARCHITECTURE AND IMPLEMENTATION}

\section{A. Architecture}

The architecture of a QRD and MMSE calculation for an $N \times N$ MIMO system is presented in Figure 8. The QRD of the matrix is calculated with an algorithm which produces a unitary matrix Q. The input matrix is either the extended channel matrix or the matrix used in the capacity and SINR calculations. The same QRD architecture can be used for both matrices. For example, the Gram-Schmidt process from [12] without column reordering can be used for the QRD. Part of the multipliers can be turned off when calculating capacity because the input matrix is smaller than the extended channel matrix. Another option is to extend the capacity/SINR matrix with zeros and then the QRD architecture can be used in exactly the same way as with the extended channel matrix. 
The capacity is obtained by multiplying the diagonal elements of $\mathbf{R}$ and taking the logarithm from the product. The SINR for stream $n$ is found by calculating the inverse of the diagonal element $(n, n)$ of matrix $\mathbf{W}$.

The decision on the modulation and coding scheme can be obtained by summing the SINR or capacity of the subcarriers in an OFDM symbol and using a lookup table to select the modulation, code rate and transmission rank based on the sum. The calculation of QRD and capacity can be time interleaved as they are not performed in each frame but in the channel coherence time. If precoding is used, multiplying the channel matrix with different precoding matrices is added to the left side of the architecture. A separate block can be added to calculate the rank 1 and 2 capacities as matrix inversion is not necessary.

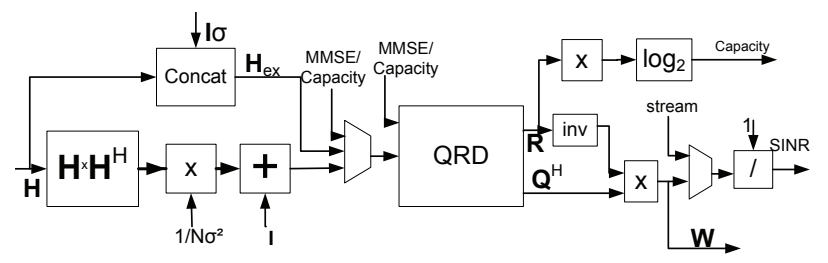

Fig. 8. Architecture of the capacity and MMSE/QRD calculation.

\section{B. Implementation results}

The LMMSE, SIC and $K$-best LSD receivers were designed to operate with an adaptive modulation scheme in a $2 \times 2$ and a $4 \times 4$ antenna system. Therefore, the receivers can be used with QPSK, 16-QAM and 64-QAM. Catapult (B) C Synthesis tool [13], which produces VHDL from $\mathrm{C}++$, was used in the implementation. The Synopsys Design Compiler was used in synthesizing the VHDL along with the $0.18 \mu \mathrm{m}$ CMOS technology. The maximum decoding rate target was set to 210 $\mathrm{Mb} / \mathrm{s}$ which is the highest rate needed for a $20 \mathrm{MHz}$ bandwidth (with 1200 used subcarriers) and 64-QAM. The decoder is not included in the complexity results but a high throughput turbo decoder [14] can be used to achieve the LTE decoding rate requirement.

The complexities in gate equivalents (GE) of the preprocessing, detection and LLR calculation are presented in Table I for the $2 \times 2$ system and Table II for the $4 \times 4$ system. The clock frequency was $150 \mathrm{MHz}$ with the $K$-best LSD and $100 \mathrm{MHz}$ with the other receivers and preprocessing. In the $2 \times 2$ case, the MMSE was calculated with the direct matrix inversion and the QRD with the Gram-Schmidt algorithm. The tree search calculation was the most complex block. The latency of the block is the time after which processing of the next input can be started. The preprocessing latency can be higher than the detection latency as it has to be performed when the channel realization changes. In the $4 \times 4$ system, the QRD is one of the most complex blocks. An $8 \times 16$ real valued extended channel matrix is used as an input to the QRD. The direct matrix inversion for MMSE weight matrix calculation would be more complex in the $4 \times 4$ system. The QRD of the extended channel matrix is used to calculate the weight matrix as presented in (6). The word lengths of the different receivers are mostly 16 bits. In the QRD, some larger inner word lengths were used and if the QRD is used for capacity calculation, the inputs are scaled as the capacity values are larger than the channel values.

TABLE I

THE $2 \times 2$ IMPLEMENTATION RESULTS

\begin{tabular}{|l|c|c|c|}
\hline Receiver Block & $\begin{array}{c}\text { Complexity } \\
(\mathrm{GE})\end{array}$ & $\begin{array}{c}\text { Power } \\
(\mathrm{mW})\end{array}$ & $\begin{array}{c}\text { Latency } \\
\mathrm{ns}\end{array}$ \\
\hline QRD (Gram-Schmidt) & $66.6 \mathrm{k}$ & 35.1 & 400 \\
\hline MMSE (direct matrix inversion) & $48 \mathrm{k}$ & 40 & 400 \\
\hline Detection and LLR & $17.1 \mathrm{k}$ & 12.8 & 10 \\
\hline SIC & $19.6 \mathrm{k}$ & 24.5 & 40 \\
\hline 4-best LSD & $67.4 \mathrm{k}$ & 101.3 & 80 \\
\hline 8-best LSD & $110.3 \mathrm{k}$ & 164 & 80 \\
\hline LLR for 4-best & $18.4 \mathrm{k}$ & 22 & 80 \\
\hline LLR for 8-best & $25.9 \mathrm{k}$ & 36.3 & 80 \\
\hline LLR it. for 8-best & $34.9 \mathrm{k}$ & 58.1 & 40 \\
\hline
\end{tabular}

TABLE II

THE $4 \times 4$ IMPLEMENTATION RESULTS

\begin{tabular}{|l|c|c|c|}
\hline Receiver Block & $\begin{array}{c}\text { Complexity } \\
\text { (GE) }\end{array}$ & $\begin{array}{c}\text { Power } \\
(\mathrm{mW})\end{array}$ & $\begin{array}{c}\text { Latency } \\
\text { ns }\end{array}$ \\
\hline QRD (Gram-Schmidt) & $179.8 \mathrm{k}$ & 116 & 4480 \\
\hline $\mathbf{R}^{-1}$ (for MMSE) & $43.5 \mathrm{k}$ & 31.9 & 3420 \\
\hline Matrix mult. (for MMSE) & $29 \mathrm{k}$ & 31.4 & 3200 \\
\hline Detection and LLR & $31 \mathrm{k}$ & 33.7 & 50 \\
\hline SIC & $86.3 \mathrm{k}$ & 88.7 & 40 \\
\hline 4-best LSD & $140.3 \mathrm{k}$ & 212.2 & 80 \\
\hline 8-best LSD & $246 \mathrm{k}$ & 387.2 & 80 \\
\hline LLR for 4-best & $21.5 \mathrm{k}$ & 29.3 & 80 \\
\hline LLR it. for 4-best & $34.5 \mathrm{k}$ & 61.6 & 40 \\
\hline LLR for 8-best & $26.4 \mathrm{k}$ & 38.7 & 80 \\
\hline
\end{tabular}

The total complexity and power consumption of the receivers are presented in Tables III and IV. The $K$-best LSD has the highest complexity. In the $2 \times 2$ system, the complexity of the SIC receiver is much lower even though the performance is close to that of the $K$-best LSD.

TABLE III

COMPLEXITY OF $2 \times 2$ RECEIVERS WITH A $210 \mathrm{MB} / \mathrm{S}$ DECODING RATE

\begin{tabular}{|c|c|c|}
\hline Receiver & $\begin{array}{c}\text { Complexity } \\
\text { (GE) }\end{array}$ & $\begin{array}{c}\text { Power consumption } \\
(\mathrm{mW})\end{array}$ \\
\hline LMMSE & $59.5 \mathrm{k}$ & 52.4 \\
\hline SIC & $84.7 \mathrm{k}$ & 77.3 \\
\hline 4-best & $152.5 \mathrm{k}$ & 158.4 \\
\hline 8-best & $197.1 \mathrm{k}$ & 223 \\
\hline 8-best, 2 it. & $206 \mathrm{k}$ & 244.8 \\
\hline
\end{tabular}

The difference in complexity between the receivers is smaller in the $4 \times 4$ case than in the $2 \times 2$ case. Calculating the MMSE weight matrix $\mathbf{W}$ takes almost 90 percent of the gate equivalents in the LMMSE receiver in the $4 \times 4$ case. The $2 \times 2$ system allowed some simplifications in the implementation and cannot be straightforwardly scaled to all other antenna configurations. The $4 \times 4$ implementation could be also scaled to larger antenna configurations.

If precoding is used, the complexity of the receiver is increased. The capacity has to be calculated for the 16 possible 
TABLE IV

COMPLEXITY OF $4 \times 4$ RECEIVERS WITH A 405 MB/S DECODING RATE

\begin{tabular}{|c|c|c|}
\hline Receiver & $\begin{array}{c}\text { Complexity } \\
\text { (GE) }\end{array}$ & $\begin{array}{c}\text { Power consumption } \\
\text { (mW) }\end{array}$ \\
\hline LMMSE & $283.3 \mathrm{k}$ & 213 \\
\hline SIC & $369.6 \mathrm{k}$ & 302 \\
\hline 4-best & $341.6 \mathrm{k}$ & 358 \\
\hline 4-best, 2 it. & $453.5 \mathrm{k}$ & 390 \\
\hline 8-best & $452.3 \mathrm{k}$ & 542 \\
\hline
\end{tabular}

precoding matrices in LTE with the different precoding matrix ranks. For rank 4 transmission, the capacity is the same for all precoding matrices and the capacity has to be calculated only once. The QRD can be used to calculate the determinant as shown in Figure 8. The matrix multiplications and the low rank determinant calculations add still another $82 \mathrm{k}$ gates to the complexity as shown in Table V. Without precoding, the capacity calculations are more simple and most of the complexity comes from the $\mathbf{H}^{\mathrm{H}} \mathbf{H}$ matrix multiplication.

TABLE V

COMPLEXITY OF PRECODING $4 \times 4$ SYSTEM

\begin{tabular}{|c|c|c|}
\hline Block & $\begin{array}{c}\text { Complexity } \\
\text { (GE) }\end{array}$ & $\begin{array}{c}\text { Power } \\
(\mathrm{mW})\end{array}$ \\
\hline Capacity with precoding & $81.9 \mathrm{k}$ & 39.2 \\
\hline Capacity without precoding & $32 \mathrm{k}$ & 36 \\
\hline
\end{tabular}

The goodput with different receivers is presented in Table VI at different SNRs and with AMC and the fixed MCS of 64-QAM and 4/5 code rate. As the decoding rates are high enough for the $20 \mathrm{MHz}$ bandwidth, the goodput depends on the performance of the receiver. The FER remains at roughly 1 percent with the AMC but with the fixed scheme, the FER increases as the signal-to-noise ratio decreases.

TABLE VI

THE GOODPUT WITH DIFFERENT SNRS

\begin{tabular}{|c|c|c|c|c|}
\hline Receiver & $\begin{array}{c}\text { AMC } \\
\text { at } 30 \mathrm{~dB}\end{array}$ & $\begin{array}{c}\text { AMC } \\
\text { at } 4 \mathrm{~dB}\end{array}$ & $\begin{array}{c}\text { Fixed MCS } \\
\text { at } 30 \mathrm{~dB}\end{array}$ & $\begin{array}{c}\text { Fixed MCS } \\
\text { at } 4 \mathrm{~dB}\end{array}$ \\
\hline LMMSE & $86.6 \mathrm{Mb} / \mathrm{s}$ & $26 \mathrm{Mb} / \mathrm{s}$ & $7.4 \mathrm{Mb} / \mathrm{s}$ & $0 \mathrm{Mb} / \mathrm{s}$ \\
\hline SIC & $114 \mathrm{Mb} / \mathrm{s}$ & $26 \mathrm{Mb} / \mathrm{s}$ & $27.7 \mathrm{Mb} / \mathrm{s}$ & $0 \mathrm{Mb} / \mathrm{s}$ \\
\hline 8 -best & $117 \mathrm{Mb} / \mathrm{s}$ & $25.5 \mathrm{Mb} / \mathrm{s}$ & $86 \mathrm{Mb} / \mathrm{s}$ & $0 \mathrm{Mb} / \mathrm{s}$ \\
\hline
\end{tabular}

\section{CONCLUSIONS}

A system with adaptive modulation and coding and the LTE based precoding was used to compare the performancecomplexity tradeoff of detection algorithms for the MIMOOFDM downlink receiver. The receivers were designed to meet the decoding rate requirements in LTE of $210 \mathrm{Mb} / \mathrm{s}$ in the $2 \times 2$ antenna system and $405 \mathrm{Mb} / \mathrm{s}$ in the $4 \times 4$ system. The complexities and goodput of the LMMSE, SIC and $K$ best receivers in a $2 \times 2$ antenna system are summarized in Table VII. At the low SNRs the performances of the receivers are similar but when the transmission rank is increased at the high SNRs, the more complex receivers give a higher goodput. A reconfigurable architecture could be used, where a low complexity receiver in the low SNRs and a more complex receiver in the high SNRs would provide the highest goodput with the least amount of power.
Different mobile velocities may have an impact on the feedback and the performance of AMC. Partial channel state information may also be utilized in the system. The scaling of the implementation to different modulation schemes is also a topic of future work.

TABLE VII

THE $2 \times 2$ PERFORMANCE-COMPLEXITY TRADEOFF

\begin{tabular}{|c|c|c|c|}
\hline Receiver & GE & Goodput at 5 dB & Goodput at 28 dB \\
\hline LMMSE & $60 \mathrm{k}$ & $28 \mathrm{Mb} / \mathrm{s}$ & $90 \mathrm{Mb} / \mathrm{s}$ \\
\hline SIC & $85 \mathrm{k}$ & $36 \mathrm{Mb} / \mathrm{s}$ & $98 \mathrm{Mb} / \mathrm{s}$ \\
\hline 8-best & $197 \mathrm{k}$ & $36 \mathrm{Mb} / \mathrm{s}$ & $114 \mathrm{Mb} / \mathrm{s}$ \\
\hline 8-best, 2 it. & $206 \mathrm{k}$ & $36 \mathrm{Mb} / \mathrm{s}$ & $126 \mathrm{Mb} / \mathrm{s}$ \\
\hline
\end{tabular}

\section{ACKNOWLEDGMENTS}

The authors would like to thank Mentor Graphics for the possibility to evaluate Catapult C B Synthesis tool.

\section{REFERENCES}

[1] H. Boelcskei, "MIMO-OFDM wireless systems: Basics, perspectives, and challenges," IEEE Trans. Commun., vol. 13, no. 4, pp. 31-37, Aug. 2006.

[2] 3rd Generation Partnership Project (3GPP); Technical Specification Group Radio Access Network, "Evolved universal terrestrial radio access E-UTRA; physical channels and modulation TS 36.211 (version 8.5.0)," Tech. Rep., 2008.

[3] M. O. Damen, H. E. Gamal, and G. Caire, "On maximum-likelihood detection and the search for the closest lattice point," IEEE Trans. Inform. Theory, vol. 49, no. 10, pp. 2389-2402, Oct. 2003.

[4] B. Hochwald and S. ten Brink, "Achieving near-capacity on a multipleantenna channel," IEEE Trans. Commun., vol. 51, no. 3, pp. 389-399, Mar. 2003.

[5] K. Wong, C. Tsui, R. K. Cheng, and W. Mow, "A VLSI architecture of a K-best lattice decoding algorithm for MIMO channels," in Proc. IEEE Int. Symp. Circuits and Systems, vol. 3, Scottsdale, AZ, May 26-29 2002, pp. 273-276.

[6] P. W. Wolniansky, G. J. Foschini, G. D. Golden, and R. A. Valenzuela, "V-BLAST: An architecture for realizing very high data rates over the rich-scattering wireless channel," in International Symposium on Signals, Systems, and Electronics (ISSSE), Pisa, Italy, Sep. 29-Oct. 2 1998, pp. 295-300.

[7] G. D. Golden, C. J. Foschini, R. A. Valenzuela, and P. W. Wolniansky, "Detection algorithm and initial laboratory results using V-BLAST space-time communication architecture," IEE Electron. Lett., vol. 35, no. 1, pp. 14-16, Jan. 1999.

[8] K. R. Kumar, G. Caire, and A. L. Moustakas, "Asymptotic performance of linear receivers in MIMO fading channels," IEEE Transactions on Information Theory, vol. 55, no. 10, pp. 4398-4418, October 2009.

[9] J. Ketonen, M. Myllylä, M. Juntti, and J. R. Cavallaro, "ASIC implementation comparison of SIC and LSD receiver for MIMO-OFDM," in Proc. Annual Asilomar Conf. Signals, Syst., Comp., Pacific Grove, USA, Oct. 25-29 2008.

[10] J. Ylioinas and M. Juntti, "Iterative joint detection, decoding, and channel estimation in turbo coded MIMO-OFDM," IEEE Trans. Veh. Technol., vol. 58, no. 4, pp. 1784-1796, May 2009.

[11] 3rd Generation Partnership Project (3GPP); Technical Specification Group Radio Access Network, "Radio transmission and reception (3G TS 45.005 version 5.4.0 (release 5))," 3rd Generation Partnership Project (3GPP), Tech. Rep., 2002.

[12] D. Wübben, R. Böhnke, J. Rinas, V. Kühn, and K. D. Kammeyer, "Efficient algorithm for decoding layered space-time codes," IEE Electronic Letters, vol. 37, no. 22, pp. 1348-1349, Oct. 2001.

[13] Mentor Graphics, "Catapult C Synthesis, Tech. Rep., http://www.mentor.com/products/esl/high_level_synthesis/catapult_synthesis," 2009.

[14] Y. Sun, Y. Zhu, M. Goel, and J. Cavallaro, "Configurable and scalable high throughput turbo decoder architecture for multiple 4G wireless standards," in IEEE Int. Conf. on Application-specific Systems, Architectures and Processors (ASAP), Leuven, Belgium, Jul.2-4 2008, pp. 209-214. 